\title{
Influence of Pixel Etching on Electrical and Electro-Optical Performances of a Ga-Free InAs/InAsSb T2SL Barrier Photodetector for Mid-Wave Infrared Imaging
}

\author{
Maxime Bouschet 1,2, Ulises Zavala-Moran 1,3, Vignesh Arounassalame ${ }^{4}$, Rodolphe Alchaar ${ }^{1}$ (D) Clara Bataillon ${ }^{1}$, \\ Isabelle Ribet-Mohamed ${ }^{4}$, Francisco de Anda-Salazar ${ }^{3}$, Jean-Philippe Perez ${ }^{1}$, Nicolas Péré-Laperne ${ }^{2}$ and \\ Philippe Christol ${ }^{1, *(D)}$
}

1 IES, Univ. Montpellier, CNRS, F-34000 Montpellier, France; bouschet@ies.univ-montp2.fr (M.B.); ulises.zavala-moran@ies.univ-montp2.fr (U.Z.-M.); alchaar@ies.univ-montp2.fr (R.A.); bataillon@ies.univ-montp2.fr (C.B.); perez@ies.univ-montp2.fr (J.P.P.)

2 LYNRED, BP 21, F-38113 Veurey-Voroize, France; Nicolas.Pere-laperne@lynred.com

3 IICO, Univ. Autónoma de San Luis Potosí, Av. Karakorum 1470, CP 78210 San Luis Potosí, Mexico; francisco.deanda@gmail.com

4 ONERA, Chemin de la Hunière, F-91761 Palaiseau, France; vignesh.arounassalame@onera.fr (V.A.); isabelle.ribet@onera.fr (I.R.-M.)

* Correspondence: christol@ies.univ-montp2.fr

Citation: Bouschet, M.; Zavala-Moran, U.; Arounassalame, V.; Alchaar, R.; Bataillon, C.;

Ribet-Mohamed, I.; de Anda-Salazar,

F.; Perez, J.-P.; Péré-Laperne, N.;

Christol, P. Influence of Pixel Etching on Electrical and Electro-Optical Performances of a Ga-Free InAs/InAsSb T2SL Barrier Photodetector for Mid-Wave Infrared Imaging. Photonics 2021, 8, 194. https://doi.org/10.3390/photonics 8060194

Received: 29 April 2021

Accepted: 28 May 2021

Published: 30 May 2021

Publisher's Note: MDPI stays neutral with regard to jurisdictional claims in published maps and institutional affiliations.

Copyright: (c) 2021 by the authors. Licensee MDPI, Basel, Switzerland. This article is an open access article distributed under the terms and conditions of the Creative Commons Attribution (CC BY) license (https:/ / creativecommons.org/licenses/by/ $4.0 /)$.

\begin{abstract}
In this paper, the influence of etching depth on the dark current and photo-response of a mid-wave infrared Ga-free T2SL XBn pixel detector is investigated. Two wet chemical etching depths have been considered for the fabrication of a non-passivated individual pixel detector having a cut-off wavelength of $5 \mu \mathrm{m}$ at $150 \mathrm{~K}$. This study shows the strong influence of the lateral diffusion length of a shallow-etched pixel on the electro-optical properties of the device. The lowest dark current density was recorded for the deep-etched detector, on the order of $1 \times 10^{-5} \mathrm{~A} / \mathrm{cm}^{2}$ at $150 \mathrm{~K}$ and a bias operation equal to $-400 \mathrm{mV}$. The corresponding quantum efficiency was measured at $60 \%$ (without anti-reflection coating) for a $3 \mu \mathrm{m}$ thick absorbing layer. A comparison of experimental results obtained on the two kinds of etched pixels demonstrates the need for a deep-etching process combined with efficient passivation for FPA manufacturing.
\end{abstract}

Keywords: mid-wave infrared quantum detector; barrier structure; Ga-free type-II superlattice; pixel etching

\section{Introduction}

In the past ten years, barrier structure (called XBn) has become the standard design of antimonide-based (Sb-based) high-performance cooled infrared (IR) photodetectors, particularly for Ga-free InAsSb bulk and InAs/InAsSb type-II superlattice (T2SL) devices operating in the mid-wave infrared (MWIR, 3-5 $\mu \mathrm{m})$ spectral domain [1-11].

The XBn barrier structure, if well designed and correctly fabricated, permits to control the dark currents in IR photodetectors. In this structure, a barrier layer (BL) made of a large band gap material is inserted between the contact layer (CL) and the n-type absorbing layer (AL) to block the electron majority carriers without impeding the transport of the hole minority carriers. Therefore, this device is also called an unipolar barrier detector. With the electric field being confined in the BL, the generation-recombination current is suppressed in the AL. Thence, the dark current of such a structure is diffusion limited whatever the temperature and, at a given dark current value, the temperature operation should be enhanced compared to the usual pin photodiode. A temperature operation higher than $140 \mathrm{~K}$ has been achieved in the MWIR domain for Ga-free T2SL XBn detector focal plane arrays (FPAs) [3-5,8,9]. Specifically, Ting et al. [4] reported a $24 \mu \mathrm{m}$ pitch, $640 \times 512$ format FPA with a cut-off wavelength of $5.4 \mu \mathrm{m}$ exhibiting a mean noise equivalent differential 
temperature (NETD) of $18.5 \mathrm{mK}$ and an NETD operability of $99.7 \%$ at a $150 \mathrm{~K}$ operating temperature with an $\mathrm{f} / 2$ aperture and a $300 \mathrm{~K}$ background. Obtaining such an operating temperature, significantly higher than the MWIR market-leading InSb technology (80 K), leads to a reduction in size, weight, and power consumption of the cryocooler of the IR imaging system [12], which would then be suitable for a new class of applications where the needs in portability, compactness and energy autonomy of the IR cameras are essential. For instance, smaller size and weight will enable higher compactness of an IR night vision goggle used by a soldier for whom each additional weight to carry means more pain and reduced agility. Less power consumption will permit to ease the miniature Unmanned Aerial Vehicle (UAV) payloads or to enhance the mission time of a satellite embedding IR imaging system for space observation.

Another important feature of the $\mathrm{XBn}$ barrier structure is the possible control of surface leakage currents [13]. Indeed, the presence of the BL, which has to be thick and high enough to suppress, respectively, the tunneling current and thermionic current of electrons coming from the $\mathrm{CL}$, blocks the flow of electrons coming from surface states due to air-exposed semiconductor surfaces. Consequently, it is possible to avoid the need for surface passivation treatments when the device is fabricated by etching up to the BL. The main advantage of such shallow etching isolation is therefore a simplified process for the fabrication of individual pixel devices. However, in the case of large-format IR FPAs with a small pixel pitch, lower than $25 \mu \mathrm{m}$, a significant lateral diffusion length of minority photo-generated carriers could induce electrical and optical pixel-to-pixel crosstalk since the pixels are not fully reticulated [14-16].

The objective of this paper is to study the influence of the etching depth of a nonpassivated pixel on electrical and electro-optical performances of an MWIR Ga-free InAs / InAsSb XBn T2SL barrier detector.

\section{Materials and Methods}

In this part, the fabrication of a Ga-free InAs/InAsSb XBn T2SL single-pixel barrier photodetector and its typical characterizations are reported.

The T2SL detector material was epitaxially grown on 2-inch n-type (Te-doped) GaSb (100) substrate by molecular beam epitaxy (MBE) using a 412 RIBER Machine. Figure 1a shows the stacking of the grown structure. From bottom to top, the $\mathrm{XBn}$ structure consists of a $400 \mathrm{~nm}$ Te-doped (n-type) GaSb buffer layer, which is followed by a $100 \mathrm{~nm}$ thick n-type doped InAs $/ \operatorname{InAs}_{0.65} \mathrm{Sb}_{0.35}$ T2SL and by non-intentionally doped (nid) $3 \mu \mathrm{m}$ thick AL (536 periods) made of the same T2SL structure. A BL is then made from $120 \mathrm{~nm}$ nid $\mathrm{AlAs}_{0.09} \mathrm{Sb}_{0.91}$, and finally the CL of the structure is composed of an $80 \mathrm{~nm}$ thick nid T2SL. The AL, CL, and BL are undoped, and the residual doping is expected to be n-type for the two first layers and p-type for the third one, at $3 \times 10^{15} \mathrm{~cm}^{-3}$ and $2 \times 10^{16} \mathrm{~cm}^{-3}$, respectively. These doping concentrations have been determined by capacitance-voltage measurements [11].

Prior to device fabrication, several material characterizations were routinely performed. They are mainly composed of a high-resolution X-ray diffraction (HR-XRD) scan to evaluate the structural quality of the XBn sample and its lattice mismatch with the GaSb substrate, atomic force microscope (AFM) images to assess the surface roughness and morphology, photoluminescence (PL) spectra to determine the bandgap energy of the T2SL structure at the targeted temperature of operation and time-resolved photoluminescence (TRPL) measurements on XBn structure to extract the minority carrier lifetime. 
(a)

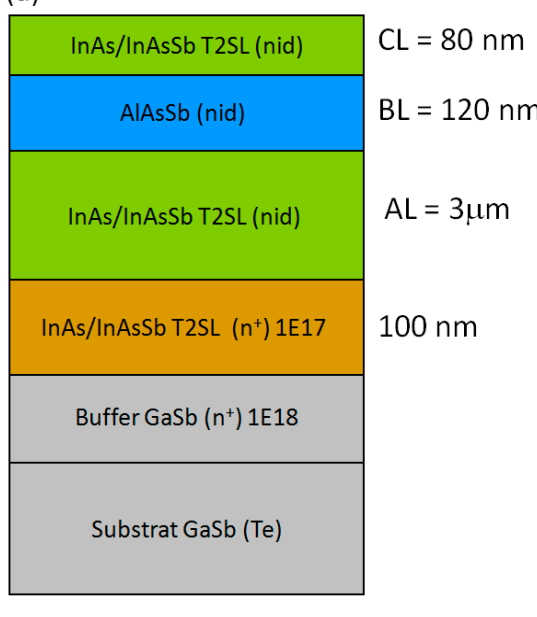

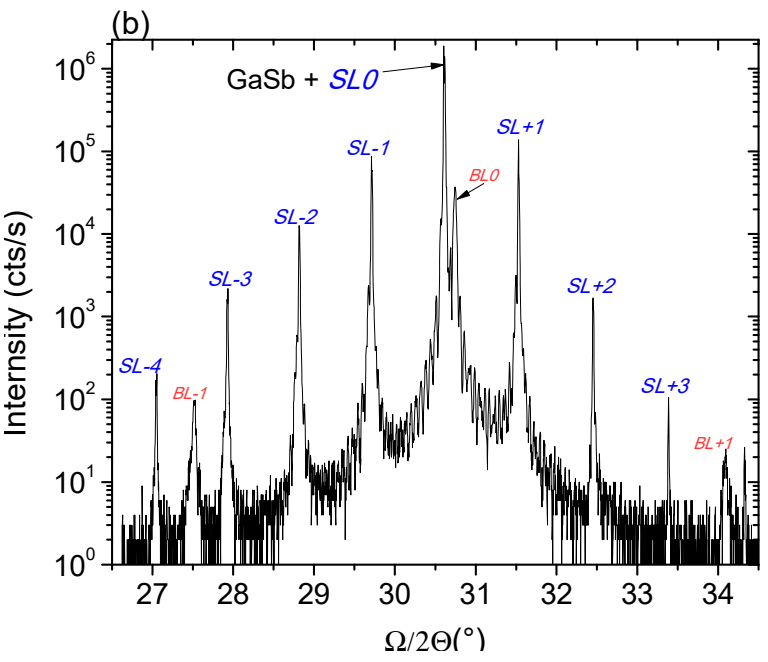

Figure 1. (a) Stacking of the XBn InAs/InAsSb T2SL structure grown by MBE on GaSb substrate. (b) HR-XRD pattern of the T2SL detector structure. The lattice mismatch with the GaSb substrate is $-354 \mathrm{ppm}$.

The experimental HR-XRD pattern of the InAs/InAsSb T2SL is shown in Figure 1b. On both sides of the 0th order peak (SL0), one can observe many intense satellite peaks (from SL-4 to SL+3) attesting to the good crystalline quality of the layers and a high reproducibility rate in the SL period. From the HR-XRD scan curve, it is possible to extract the composition of each layer. The AL is composed of an InAs $(4.17 \mathrm{~nm}) / \mathrm{InAs}_{0.658} \mathrm{Sb}_{0.342}(1.42 \mathrm{~nm}) \mathrm{T} 2 \mathrm{SL}$ while the AlAsSb BL, grown by an adapted digital alloy technique [17], is made of AlSb $(1.24 \mathrm{~nm}) / \mathrm{AlAs}_{0.51} \mathrm{Sb}_{0.49}(0.32 \mathrm{~nm})$ short period $\mathrm{SL}$, slightly lattice-mismatched with the $\mathrm{GaSb}$ substrate. The mismatch between the $\mathrm{AL}$ and the $\mathrm{GaSb}$ substrate is $-354 \mathrm{ppm}$ (compressive strain), while the BL shows a negative mismatch of $+4110 \mathrm{ppm}$ (tensile strain).

The other material characterization measurements have already been reported elsewhere [11], except the TRPL measurement performed on the whole XBn structure, for which a minority carrier lifetime value of $850 \mathrm{~ns}$ was extracted at $\mathrm{T}=150 \mathrm{~K}$ (Figure 2). For this measurement, a pulsed laser at $1.55 \mu \mathrm{m}$ (full width at half maximum equal to $5 \mathrm{~ns}$ ) is used to generate the PL signal, which is collected with reflective optics and focused on an $\mathrm{HgCdTe}$ photodiode with a response time constant lower than $50 \mathrm{~ns}$. The PL decays are sampled by a digital scope and averaged. The minority carrier lifetime is extracted by fitting the experimental signal by an exponential decay [18].

Next, if the material characterizations are consistent with the defined structural and optical criteria [11], the devices are processed. From epitaxial T2SL structures, circular mesa photodetectors with diameters ranging from 60 to $310 \mu \mathrm{m}$ were fabricated using standard photolithography techniques. Mesa photodetectors were realized by isotropic wet chemical etching using a citric acid solution and any specific surface treatment was used. No active passivation process has been applied on mesa sidewalls. Only a polymerized photoresist was spun after the wet etching to protect the mesa surface from ambient air. Metal coatings were applied on top of the mesa (n-type T2SL cap layer) and on the back of the n-type $\mathrm{GaSb}$ substrate, in Ti/ $\mathrm{Au}(40 / 150 \mathrm{~nm})$ and $\mathrm{Pd} / \mathrm{AuGeNi}(5 / 200 \mathrm{~nm})$, respectively.

To study the influence of etching depth on electrical and electro-optical performances of an XBn Ga-free T2SL photodetector, two kinds of single-pixel devices were fabricated: shallow-etched pixel down to the BL and a deep-etched single device below the AL (Figure 3). 


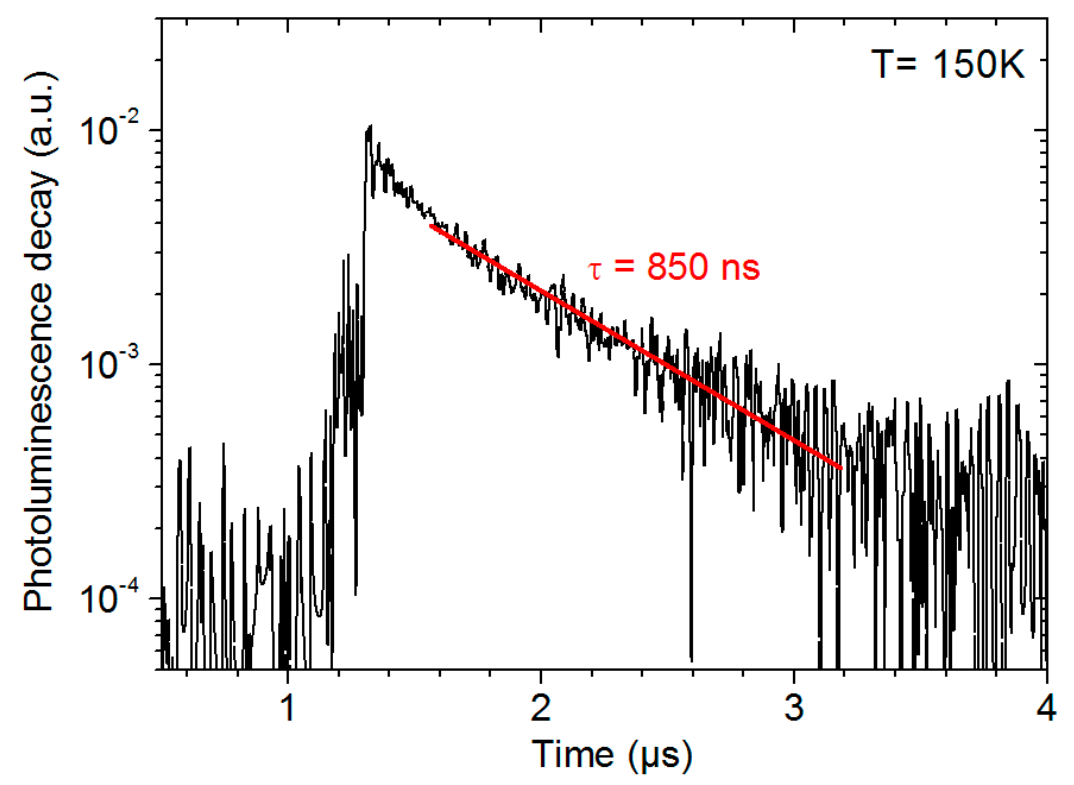

Figure 2. TRPL signal of the Ga-free $\mathrm{XBn}$ InAs/InAsSb T2SL structure at $150 \mathrm{~K}$. Following the approach of Donetsky et al. [18], a minority carrier lifetime of $850 \mathrm{~ns}$ is extracted from measurement.

a) Shallow-etched pixels

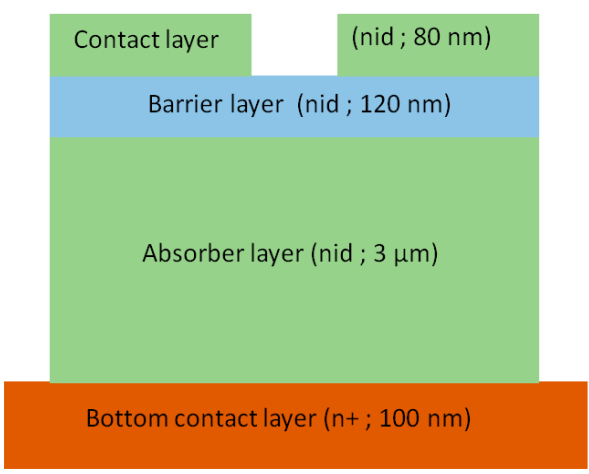

b) Deep-etched pixels

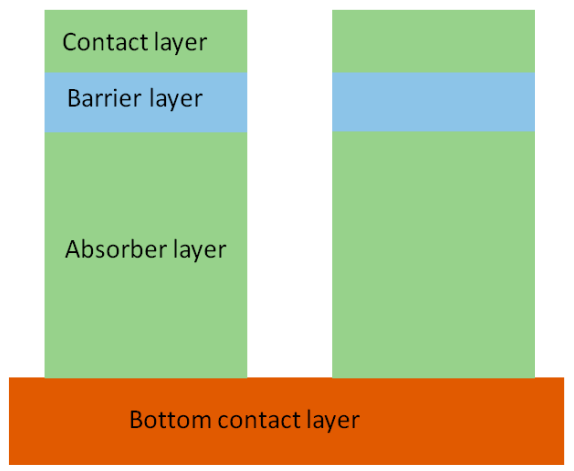

Figure 3. Schematic view of shallow-etched (a) and deep-etched (b) pixels.

The barrier detector devices were then characterized by electrical and electro-optical measurements. For that, the T2SL devices were placed in a probe station to perform dark current density-voltage (J-V) measurements (under a 0-degree field of view) for different operating temperatures. For that, a KEITHLEY 6517A Electrometer was used to both apply the bias voltage and read the current density (ratio of current and area of the device) delivered by the device.

Next, the samples were wire bonded onto a pin LCC and placed in a LN2-cooled JANIS cryostat. To determine the cut-off wavelength of the detector, the relative spectral photoresponse (PR) of the device, under front-side illumination, was measured as a function of bias and temperature using a Fourier transform infrared (FTIR) spectrometer. Figure 4 shows non-calibrated PR spectra performed at $150 \mathrm{~K}$ at operating bias $\mathrm{V}=-400 \mathrm{mV}$ and the photoluminescence (PL) spectrum.

The cut-off wavelength $\left(\lambda_{\mathrm{co}}\right)$ equal to $5 \mu \mathrm{m}$ is extracted by comparison with the PL spectrum recorded at the same temperature. It also fits the $50 \%$ cut-off wavelength approach. 


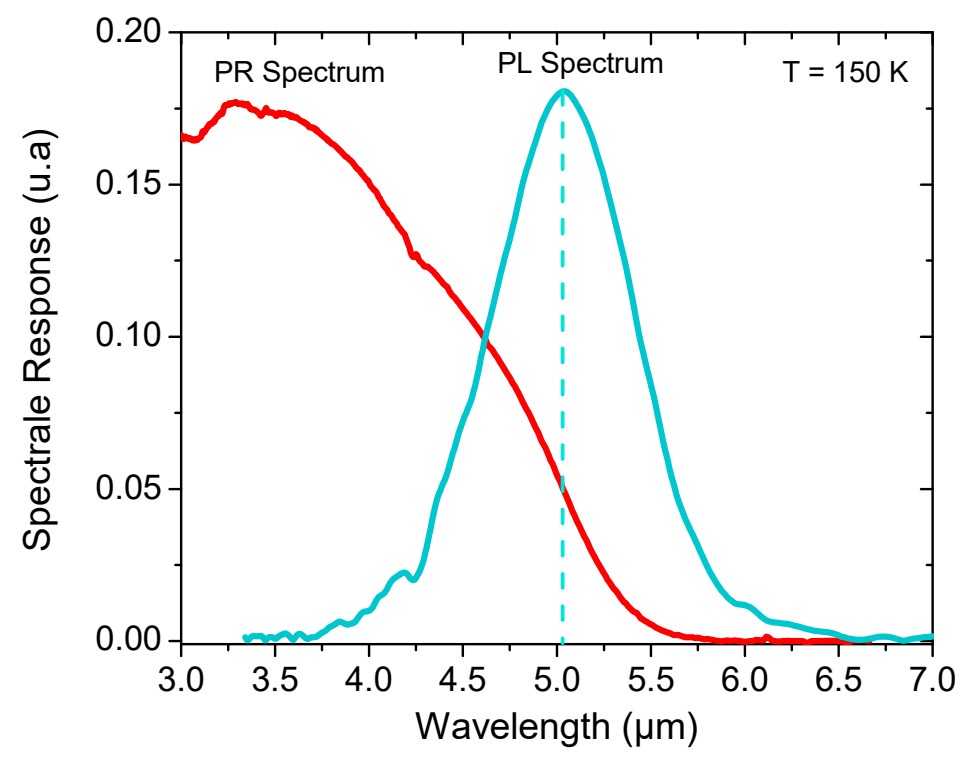

Figure 4. InAsSb/InAs T2SL structure: photo-response (PR) spectrum at $-400 \mathrm{mV}$ and photoluminescence (PL) measurement performed at $150 \mathrm{~K}$.

Finally, the quantum efficiency $(\mathrm{QE})$ is calculated by placing the photodetectors in front of a blackbody source with temperatures ranging from 973 to $1173 \mathrm{~K}$. A narrow band filter $(3 \mu \mathrm{m} ; 3.25 \mu \mathrm{m})$ is used to have a fully calibrated measurement of the photocurrent at each blackbody's temperature. The QE is then extracted from the slope of the photocurrent versus the incident flux [19].

The study on shallow- and deep-etched T2SL detector pixels was conducted from dark J-V and QE measurements.

\section{Results and Discussion}

\subsection{Shallow-Etched Photodetector}

At the expected temperature operation $\mathrm{T}=150 \mathrm{~K}, \mathrm{~J}-\mathrm{V}$ measurements in dark conditions were performed on photodetectors with different circular mesa diameter sizes ranging from 60 to $210 \mu \mathrm{m}$ and the results are shown in Figure 5a. Extracted from J-V curves, the $R_{d} A$ product is plotted in Figure $5 b$, where $R_{d} A$ represents the differential resistance, calculated from the derivative of the voltage over the current and $\mathrm{A}$ is the device area. By examining the shape of the displayed curves, we can identify two typical reverse biases. The first one, Von (turn on bias), is located at the first $R_{d} A$ minimum at $-200 \mathrm{mV}$. At this bias, thermal-generated holes can reach the CL. Next, the $R_{d} A$ peak at $-400 \mathrm{mV}$, equal to $2.7 \times 10^{4} \Omega \cdot \mathrm{cm}^{2}$, corresponds to the second one, $V_{\text {op }}$ (operating bias), for which the device is fully turned on at this bias and the photo-response value should be maximal. Consequently, it should not be necessary to increase the polarization beyond $V_{\text {op }}$ to have the highest quantum efficiency value. At $\mathrm{V}_{\mathrm{op}}$, the lowest recorded dark current density is firstly equal to $3.5 \times 10^{-5} \mathrm{~A} / \mathrm{cm}^{2}$.

The J-V curves are slightly dispersed (Figure $5 a$ ) and the dark current density was extracted at $\mathrm{V}_{\text {op }}$ and plotted versus the perimeter-to-area ratio of the device (Figure 5c). The surface dependence of the total dark current density $\left(J_{\text {total }}\right)$ is given by the following Equation (1):

$$
J_{\text {total }}=J_{\text {bulk }}+\frac{P}{A} \cdot J_{\text {surface }}
$$

where $J_{\text {bulk }}$ and $J_{\text {surface }}$ are the bulk and surface dark current density contribution, respectively, $P$ is the diode's perimeter, and $A$ is the diode's area. 

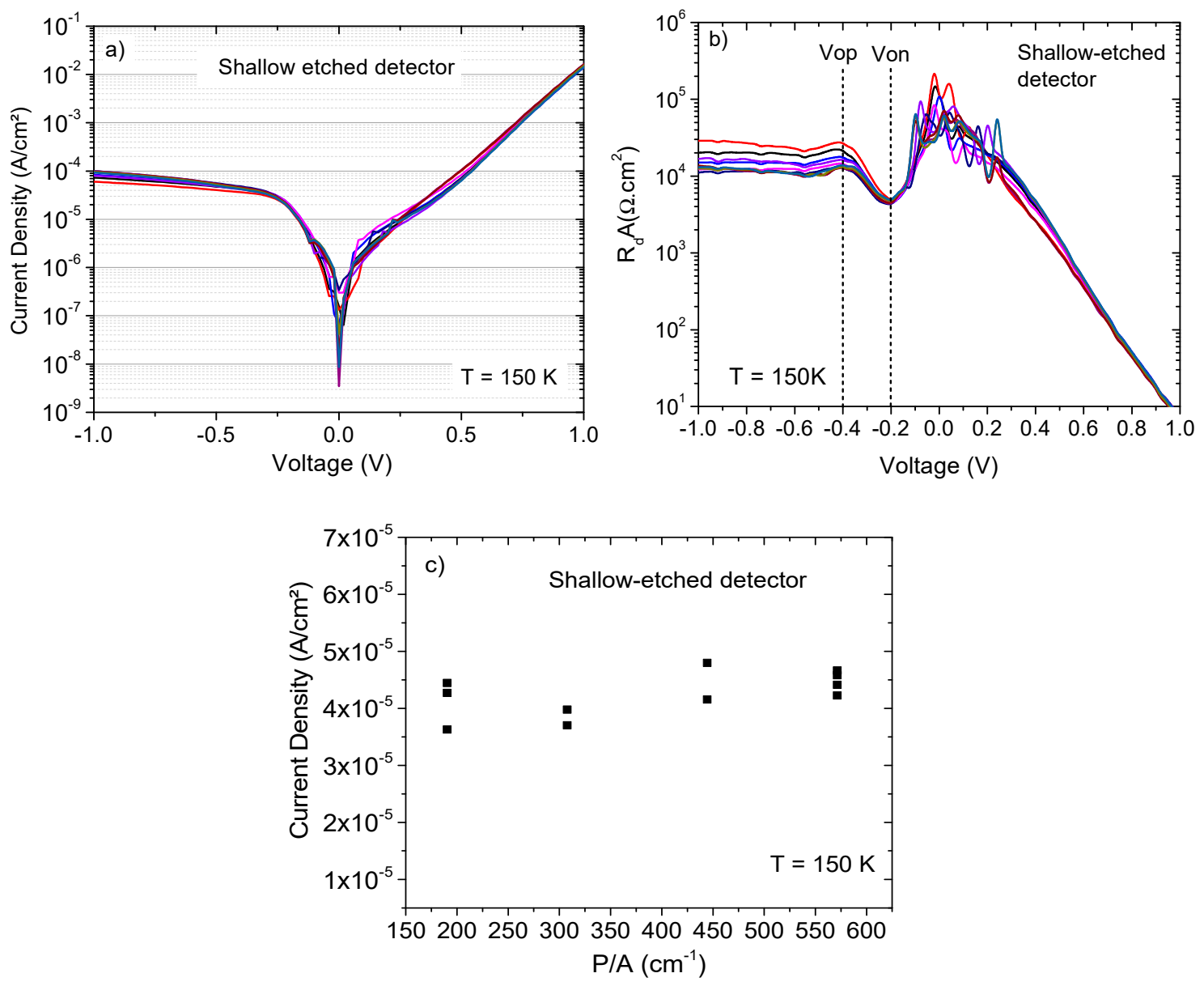

Figure 5. Shallow-etched photodetectors: (a) dark current density voltage characteristics performed on different detector sizes at $150 \mathrm{~K} ;(\mathbf{b})$ differential Resistance Area product $\left(\mathrm{R}_{\mathrm{d}} \mathrm{A}\right)$ product as a function of the voltage at $150 \mathrm{~K}$; (c) dark current density value as a function of the perimeter to area ratio $(\mathrm{P} / \mathrm{A})$, extracted at $\mathrm{V}_{\mathrm{op}}=-400 \mathrm{mV}$.

The resulting curve exhibits no size dependence. Since the devices were shallow etched, there is no leakage current and strong homogeneity in terms of dark current density. However, minority carriers can diffuse laterally to the electrical contacts from outside the etched mesa. Thus, the size of the device is defined by both the lateral diffusion length of holes and the etched dimensions, which may lead to an overestimation of the dark current density. Consequently, minority carrier lateral diffusion has to be investigated.

To estimate the lateral diffusion length from electrical measurements, we have applied in Figure 6 the method used by Plis et al. [20]. If lateral diffusion is present, every mesa detector has the additional surface area $A_{s}$ around the periphery of the detector which, in the case of circular mesas, can be expressed as:

$$
A_{s}=\pi\left(r+L_{D}\right)^{2}-\pi r^{2}
$$

where $r$ is the etched mesa radius and $L_{D}$ is the diffusion length of minority carriers (holes in the present case). The inset of Figure 6 schematically shows the circular mesa diode of radius $r$ and additional surface area $A_{s}$ around it.

Since smaller-area diodes are more dependent on surface effect (i.e., lateral diffusion current), $L_{D}$ could be estimated in the limit $\mathrm{r} \rightarrow 0$ as $L_{D}=\sqrt{\frac{A_{s}}{\pi}}$. Thus, we estimate an additional area $A_{S}=1.46 \times 10^{-5} \mathrm{~cm}^{2}$ from the intersection of the fitted line with the $x$-axis, then a lateral diffusion length of $21.5 \mu \mathrm{m}$ is deduced at $150 \mathrm{~K}$, comparable to hole diffusion length values reported in MWIR devices [20-22] at this temperature. Hence, due to the 
additional surface area created by the important lateral diffusion length, a correction in the photodetector area is necessary to obtain the real electric performance.

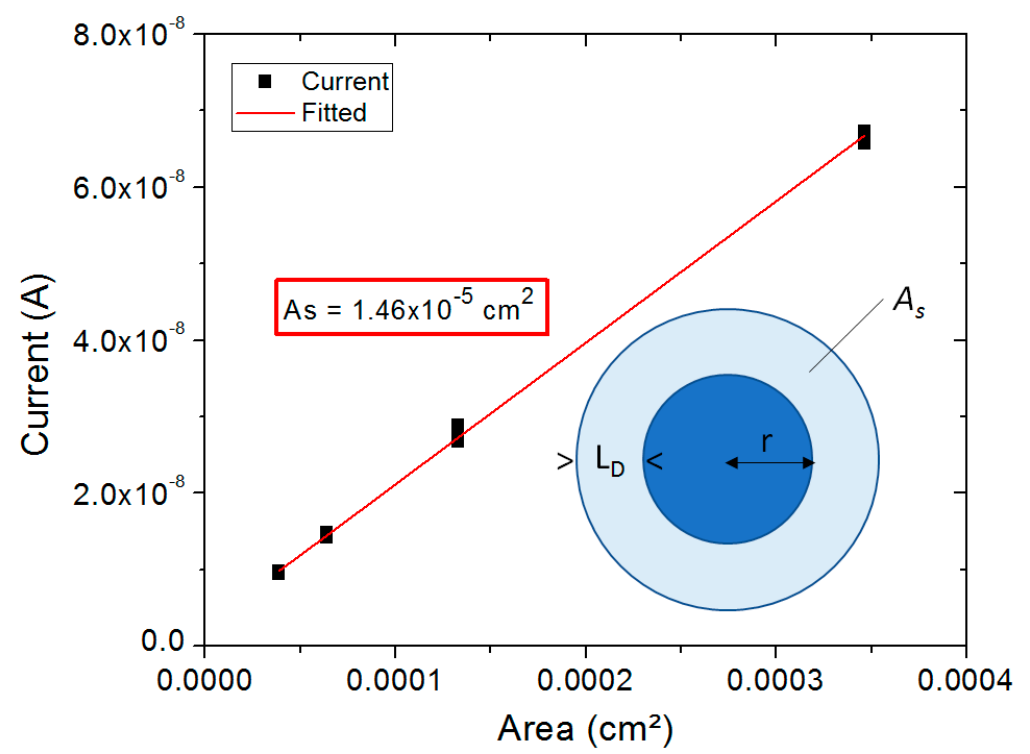

Figure 6. Dark current's dependence on the shallow-etched mesa area at $\mathrm{T}=150 \mathrm{~K}$. Inset shows the view of the circular mesa diode with the additional surface area $A_{S}$ around it due to additional lateral diffusion length $L_{D}$.

Consequently, the dark current measurements displayed in Figure 5a have to be divided by an effective surface area that included the lateral diffusion length. A dark current density value as low as $1 \times 10^{-5} \mathrm{~A} / \mathrm{cm}^{2}$ at $150 \mathrm{~K}$ is finally recorded at the operating bias $\left(\mathrm{V}_{\mathrm{op}}=-400 \mathrm{mV}\right)$.

Considering the additional surface area of the shallow-etched detector with a $210 \mu \mathrm{m}$ mesa diameter, spectral responsivity measurements, under front-side illumination, were performed at $150 \mathrm{~K}$. Figure 7 shows the spectral quantum efficiency (QE) at reverse bias ranging from $-0.15 \mathrm{~V}$ to $-0.8 \mathrm{~V}$. The non-zero operating bias value, required to turn on the full spectral response, is probably due to the presence of a valence band offset (VBO) at the barrier and absorber layers interface, blocking the transport of holes to the CL [23]. The QE starts to saturate at $-400 \mathrm{mV}$, confirming the $\mathrm{V}_{\text {op }}$ value deduced in Figure $5 \mathrm{~b}$. Concerning the increment of the QE with the bias, this behavior is due to the increase in depletion width within the $\mathrm{AL}$, allowing for a larger collection of carriers assisted by the electric field. Without anti-reflection coating, $\mathrm{QE}$ values as high as $60 \%$ at $\lambda=3.3 \mu \mathrm{m}$ were obtained, in agreement with the state-of-the-art $\mathrm{QE}$ values $[3,6,8,9]$. These QE values could be enhanced by the optimization of the electron and hole wavefunction overlap associated to the fundamental conduction and valence minibands of the T2SL structure by reducing its period thickness while increasing the antimony composition of the InAs/InAsSb heterostructure and keeping the target $5 \mu \mathrm{m}$ wavelength cut-off [24].

Finally, dark current density was measured at different temperatures, from 90 to $270 \mathrm{~K}$, and Figure 8a shows an example of dark J-V characteristics carried out for a $210 \mu \mathrm{m}$ diameter detector. The observed shift at zero bias for temperatures below $120 \mathrm{~K}$ is due to the presence of a photonic current regime in our experimental cryogenic probe station set-up. 


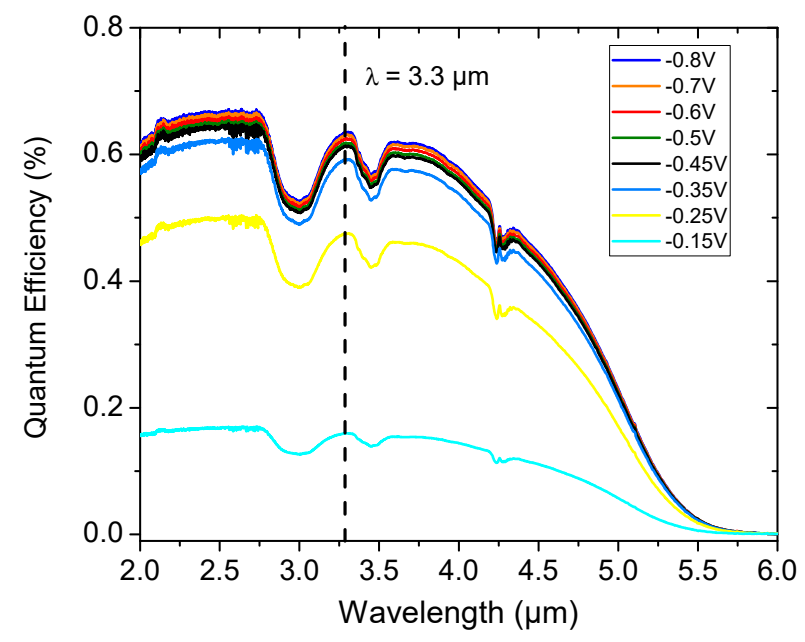

Figure 7. Front-side illuminated spectral quantum efficiency taken at $\mathrm{T}=150 \mathrm{~K}$ and under different biases of the shallow-etched circular mesa photodetectors with diameter size including the additional lateral diffusion length.

Extracted from $\mathrm{J}-\mathrm{V}$ measurements, dark current densities at operating bias $\left(\mathrm{V}_{\mathrm{op}}=\right.$ $-0.4 \mathrm{~V}$ ) are reported as a function of inverse temperature (Arrhenius plot) in Figure $8 \mathrm{~b}$. At high temperatures, between 270 and $190 \mathrm{~K}$, the diffusion dark current is dominant. In this temperature range, an activation energy $\mathrm{Ea}=0.29 \mathrm{eV}$, higher than the expected band gap energy value $\mathrm{Eg}$ (close to $0.25 \mathrm{eV}$ at $150 \mathrm{~K}$ ), is deduced from the curve. This higher energy value than the bandgap confirms the presence of a VBO at the absorber-barrier interface [23]. Such a VBO equal to $40 \mathrm{meV}$ hampers the transport of minority carriers from the $\mathrm{AL}$ to the $\mathrm{CL}$ contact layer, and an applied bias at least equal to $-0.4 \mathrm{~V}$ is needed to delete it. However, this applied bias can generate depletion in the AL and increase generation-recombination (G-R) dark current. The G-R dark current is predominant at a temperature lower than $160 \mathrm{~K}$ with an extracted activation energy equal to half bandgap energy plus the $\mathrm{VBO}$ value. At the targeted temperature operation of $150 \mathrm{~K}$, the device's design is not optimized with the presence of a depletion zone in the AL leading to a G-R dark current regime. To reduce the dark current value and perfectly suppress the G-R dark current at $150 \mathrm{~K}$, two means of improvement can be considered. One can slightly increase the doping value of the absorber layer to better confine the electric field within the BL. Another way is to reduce the VBO between the AL and BL by optimizing both the barrier composition and thickness to reduce the $V_{o p}$ and therefore the depletion within the absorber layer.
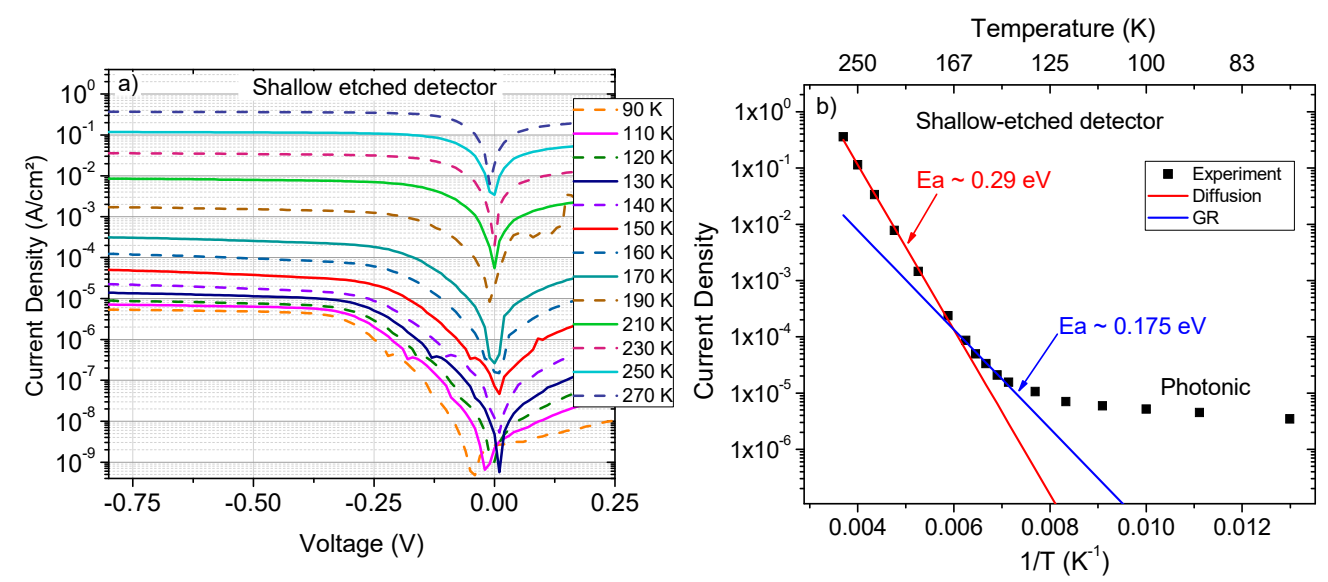

Figure 8. (a) Dark current density measured for a shallow-etched device with a $210 \mu \mathrm{m}$ diameter at different temperatures; (b) temperature dependence of dark current density at $-0.4 \mathrm{~V}$. 
In this part, we have seen that the shallow-etched detector induces an additional surface area due to lateral diffusion length. This additional effective surface area of the pixel overestimates the dark current density value but mainly can generate optical or electrical pixel-to-pixel crosstalk in an FPA imaging system. Thus, deep etching, throughout the entire absorber layer thickness, is necessary to suppress this problem and obtain the unambiguous electrical performance of the device.

\subsection{Deep-Etched Photodetector}

The deep-etched diodes used in this section were fabricated from the same epitaxial wafer as shallow-etched ones. Circular mesas were fabricated by wet etching throughout the entire absorber layer (around $3 \mu \mathrm{m}$ beneath the barrier layer), as described in Figure $3 \mathrm{~b}$.

Figure 9 presents QE values measured on the deep-etched photodiode $(210 \mu \mathrm{m}$ mesa diameter) at $150 \mathrm{~K}$ for different applied biases. Without surface area correction, a QE as high as $60 \%$ at $150 \mathrm{~K}$ is observed, similar to the one of the corrected shallow-etched device. Therefore, the etching through the total thickness of the AL allows for the electrical and electro-optical isolation of each pixel with its effective surface area. An increase in the QE with the temperature, from $47 \%$ at $90 \mathrm{~K}$ to $62 \%$ at $220 \mathrm{~K}$, is observed. This QE increment with temperature is due to the increase mobility of minority carriers and therefore in vertical diffusion length at temperatures higher than $90 \mathrm{~K}[6,21,25]$.

Furthermore, a slope of the QE with the applied bias can be observed at $90 \mathrm{~K}$. This slope is a signature of a non-optimized device at the considered temperature. Indeed, if the AL thickness is longer than the vertical diffusion length of the minority carriers, some of the photo-generated carriers recombine before reaching the CL. Thus, an increase in the depletion width with the bias is necessary to promote the full collection of minority carriers. However, the QE saturates at a higher temperature. Indeed, combining minority carrier lifetime and mobility values in Ga-free T2SL structure [6,25], the vertical diffusion length increases with the temperature, allowing for a better collection of photo-generated carriers. At $150 \mathrm{~K}$, a slight QE slope remains and, at $220 \mathrm{~K}$, the QE completely saturates over the operating bias $\left(\mathrm{V}_{\mathrm{op}}=-0.4 \mathrm{~V}\right)$ with a value higher than $60 \%$, showing that the transport of minority carriers is optimized at this temperature.

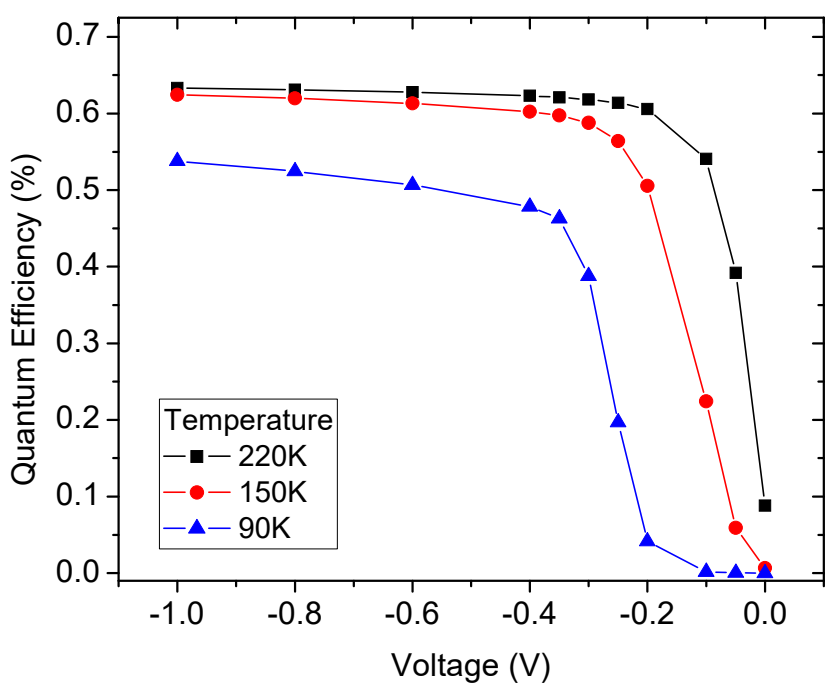

Figure 9. Quantum efficiency (QE) at $\lambda=3.4 \mu \mathrm{m}$ of the deep-etched photodetector for $\mathrm{T}=90,150$ and $220 \mathrm{~K}$ versus applied bias. At $\mathrm{T}=150 \mathrm{~K}$, the QE is equal to $60 \%$ at the operating bias $(\mathrm{V}=-0.4 \mathrm{~V})$.

Concerning electrical characterizations, Figure 10 shows the dark current density characteristics of a deep-etched detector, for temperatures ranging from 77 to $230 \mathrm{~K}$.

As for the shallow-etched device, the temperature dependence of the dark current reveals that the XBn detector is diffusion limited at high temperatures, while it is G-R limited below $160 \mathrm{~K}$. However, whatever the dark current regime, the thermal activation 
energy of the deep-etched diode is always lower than that of the shallow-etched one. Because this divergence cannot be due to a difference of VBO between shallow- and deepetched configurations, a possibility could be the variation of the considered additional surface area with the temperature and the depth etching. Complementary investigations are planned to study other pixel etching depths from the BL to the end of the AL.
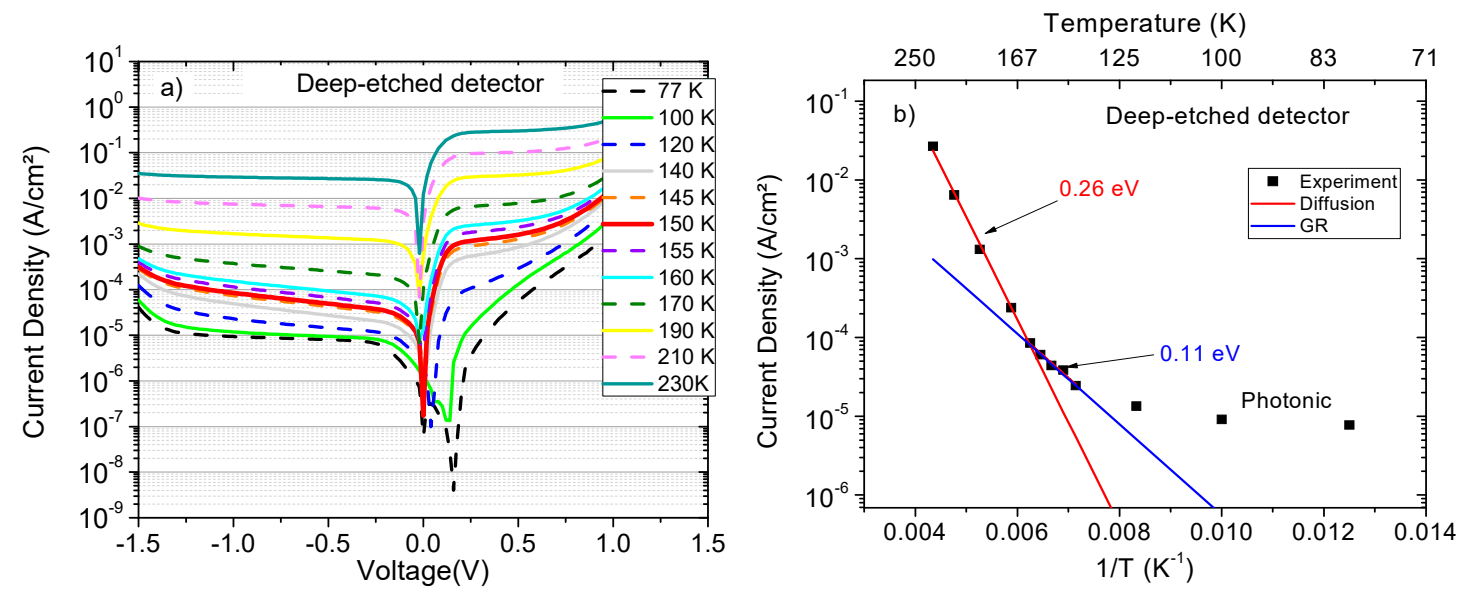

Figure 10. (a) Dark current density characteristics measured for a deep-etched device (mesa size $210 \mu \mathrm{m}$ ) at different temperatures from 77 to $230 \mathrm{~K}$; (b) temperature dependence of dark current density at $-0.4 \mathrm{~V}$.

Finally, Figure 11a shows dark current density as a function of applied bias at $150 \mathrm{~K}$ of deep-etched detectors with different sizes ranging from 60 to $210 \mu \mathrm{m}$, and Figure 11b reports the value extracted at $-400 \mathrm{mV}$ as a function of $\mathrm{P} / \mathrm{A}$ ratio. Compared to the shallow-etched device, the results are much more dispersed but the deep etching seems not to degrade the electrical performance. Indeed, dark current densities vary from $1 \times$ $10^{-5} \mathrm{~A} / \mathrm{cm}^{2}$ to $6 \times 10^{-5} \mathrm{~A} / \mathrm{cm}^{2}$ at $150 \mathrm{~K}$. A dark current density as low as $1 \times 10^{-5} \mathrm{~A} / \mathrm{cm}^{2}$ is only 7.5 times higher than the one extracted from the MCT Rule 07 [26] and such a value is among the lowest dark current density values recently reported [3,6,8,9]. However, for some devices, forward bias characteristics clearly show a signature of surface leakage current and, combined with the dispersion of results, the deep-etching process seems therefore less robust and less homogeneous than the shallow-etching one. Consequently, the deep-etching process of sidewalls sometimes degrades the electrical behavior of the pixel detector. An efficient passivation procedure is required for the fabrication of pixels, particularly in the case of high-format FPA for which the isolation of small pixels is necessary.
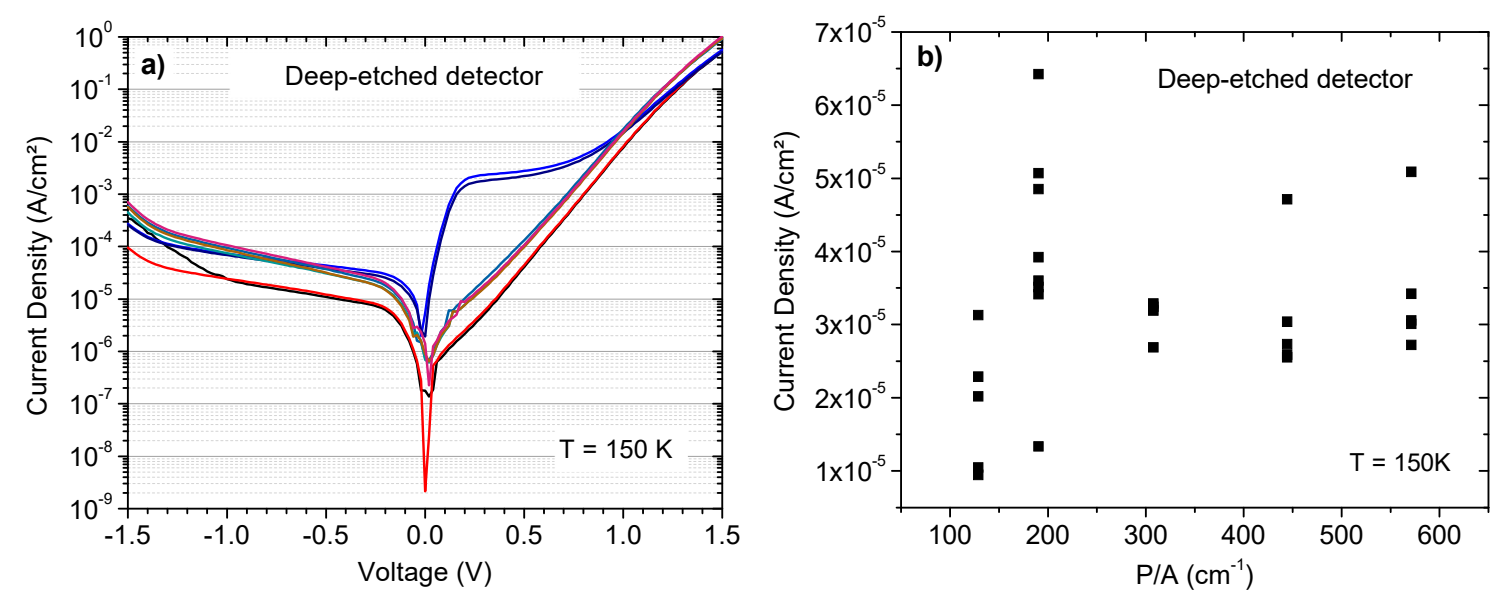

Figure 11. Deep-etched photodetectors: (a) dark current density voltage characteristics performed on different detector sizes at $150 \mathrm{~K} ;(\mathbf{b})$ dark current density value as a function of the perimeter to area ratio, extracted at $\mathrm{V}=-400 \mathrm{mV}$. 


\section{Conclusions}

We studied the influence of etching depth on electrical and electro-optical performances of a Ga-free T2SL XBn MWIR photodetector. Two kinds of wet chemical etching were investigated: a shallow-etched detector with etching just before the barrier layer; a deep-etched device with etching all over the active layer.

For both detectors showing the cut-off wavelength at $5 \mu \mathrm{m}$ at $150 \mathrm{~K}$, very good results were obtained with the same electrical and electro-optical performances: a dark current density as low as $1 \times 10^{-5} \mathrm{~A} / \mathrm{cm}^{2}$ at operating bias $(\mathrm{V}=-0.4 \mathrm{~V})$ and a quantum efficiency as high as $60 \%$ at $150 \mathrm{~K}$. These characterization values are at the state of the art, while the device is not fully optimized with a G-R limited dark current at $150 \mathrm{~K}$ and a high operating bias value due to the presence of a valence band offset. The design of the XBn detector, in terms of doping and thickness of each layer, has to be improved.

This study shows that a lateral diffusion length, inducing an additional surface area, has to be considered in the case of shallow-etched pixels, prohibiting the use of this etching for the fabrication of large-format FPAs with a very small pixel pitch. The deep-etched configuration allows one to isolate each pixel, but an important dispersion of results on dark current is observed, indicating the need for efficient sidewall surface passivation to reach good uniformity and homogeneity. The fabrication and characterization of deeply etched passivated pixels for large-format FPA imaging will be the subject of forthcoming studies.

Author Contributions: M.B., U.Z.-M., R.A. and J.-P.P. fabricated the structures and devices; M.B., U.Z.-M., R.A., C.B. and V.A. performed the measurements; M.B., J.-P.P., I.R.-M., F.d.A.-S., N.P.-L. and P.C. analyzed the data; M.B. and P.C. wrote the paper. All authors have read and agreed to the published version of the manuscript.

Funding: This work was partially funded by the French "Investment for the Future" program (EquipEx EXTRA, ANR 11-EQPX-0016) and by the French ANR under project HOT-MWIR (ANR-18CE24-0019-01).

Data Availability Statement: The data that support the findings of this study are available from the corresponding author upon reasonable request.

Conflicts of Interest: The authors declare no conflict of interest.

\section{References}

1. Maimon, S.; Wicks, G.W. nBn detector, an infrared detector with reduced dark current and higher operation temperature. Appl. Phys. Lett. 2006, 89, 151109. [CrossRef]

2. Klipstein, $\mathrm{P}$. “XBn” barrier photodetectors for high sensitivity and high operating temperature infrared sensors. In Proceedings of the SPIE Defense and Security Conference, Orlando, FL, USA, 16-21 March 2008; Volume 6940, p. 69402U.

3. Ting, D.Z.; Soibel, A.; Khoshakhlagh, A.; Rafol, S.B.; Keo, S.A.; Höglund, L.; Fisher, A.M.; Luong, E.M.; Gunapala, S.D. Midwavelength high operating temperature barrier infrared detector and focal plane array. Appl. Phys. Lett. 2018, $113,021101$. [CrossRef]

4. Ting, D.Z.; Rafol, S.B.; Keo, S.A.; Nguyen, J.; Khoshakhlagh, A.; Soibel, A.; Höglund, L.; Fisher, A.M.; Luong, E.M.; Mumolo, J.M.; et al. InAs/InASb Type-II superlattice mid-wavelength infrared focal plane array with significantly higher operating temperature than InSb. IEEE Photonics J. 2018, 10, 6804106. [CrossRef]

5. Soibel, A.; Ting, D.Z.; Rafol, S.B.; Fisher, A.M.; Keo, S.A.; Khoshakhlagh, A.; Gunapala, S.D. Mid-wavelength infrared InAsSb/InAs nBn detectors and FPAs with very low dark current density. Appl. Phys. Lett. 2019, 114, 161103. [CrossRef]

6. Soibel, A.; Ting, D.Z.; Fisher, A.M.; Khoshakhlagh, A.; Pepper, B.; Gunapala, S.D. Temperature dependence of diffusion length and mobility in mid-wavelength InAs/InAsSb superlattice infrared detectors. Appl. Phys. Lett. 2020, 117, 231103. [CrossRef]

7. Michalczewski, K.; Tsai, T.Y.; Martyniuk, P.; Wu, C.H. Demonstration of HOT photoresponse of MWIR T2SLs InAs/InAsSb photoresistors. Bull. Pol. Acad. Sci. Tech. Sci. 2019, 67, 141-145.

8. Ariyawansa, G.; Joshua Duran, J.; Reyner, C.; Scheihing, J. InAs/InAsSb strained-layer superlattice mid-wavelength infrared detector for high-temperature operation. Micromachines 2019, 10, 806. [CrossRef]

9. Deng, G.; Chen, D.; Yang, S.; Yang, C.; Yuan, J.; Yang, W.; Zhang, Y. High operating temperature pBn barrier mid-wavelength infrared photodetectors and focal plane array based on InAs/InAsSb strained-layer superlattices. Opt. Express 2020, 28, 1761117619. [CrossRef] 
10. Wu, D.; Li, J.; Dehzangi, A.; Razeghi, M. Mid-wavelength infrared high operating temperature pBn photodetectors based on type-II InAs/InAsSb superlattice. AIP Adv. 2020, 10, 025018. [CrossRef]

11. Zavala-Moran, U.; Bouschet, M.; Perez, J.P.; Alchaar, R.; Bernhardt, S.; Ribet-Mohamed, I.; De Anda-Salazar, F.; Christol, P. Structural, optical and electrical characterizations of midwave infrared Ga-free Type-II InAs/InAsSb superlattice barrier photodetector. Photonics 2020, 7, 76. [CrossRef]

12. Manissadjian, A.; Rubaldo, L.; Rebeil, Y.; Kerlain, A.; Brellier, D.; Mollard, L. Improved IR detectors to swap heavy systems for SWaP. In Proceedings of the SPIE Infrared Technology and Applications XXXVIII, Baltimore, MD, USA, 31 May 2012; Volume 8353, p. 835334.

13. Savich, G.R.; Pedrazzani, J.R.; Maimon, S.; Wicks, G.W. Use of epitaxial unipolar barriers to block surface leakage currents in photodetectors. Phys. Status Solidi C 2010, 7, 2540-2543. [CrossRef]

14. Nolde, J.A.; Jackson, E.M.; Bennett, M.F.; Affouda, C.A.; Cleveland, E.R.; Canedy, C.L.; Vurgaftman, I.; Jernigan, G.G.; Meyer, J.R.; Aifer, E.H. Reticulated shallow etch mesa isolation for controlling surface leakage in GaSb-based infrared detectors. Appl. Phys. Lett. 2017, 111, 051102.

15. Du, X.; Savich, G.R.; Marozas, B.T.; Wicks, G.W. Effects of epitaxial structure and processing on electrical characteristics of InAs-based nBn infrared detectors. In Proceedings of the SPIE Infrared Technology and Applications XLIII, Anaheim, CA, USA, 9-13 April 2017; Volume 10177, p. 101771Q.

16. Höglund, L.; Rodriguez, J.B.; Marcks von Würtemberg, R.; Naureen, S.; Ivanov, R.; Asplund, C.; Alchaar, R.; Christol, P.; Rouvié, A.; Borcal, J.; et al. Influence of shallow versus deep etching on dark current and quantum efficiency in InAs/GaSb superlattice photodetectors and focal plane arrays for long wavelength infrared detection. Inf. Phys. Technol. 2018, 95, 158-163. [CrossRef]

17. Schuler-Sandy, T.; Klein, B.; Casias, L.; Mathews, S.; Kadlec, C.; Tian, Z.; Plis, E.; Myers, S.; Krishna, S. Growth of InAs-InAsSb SLS through the use of digital alloys. J. Cryst. Growth 2015, 425, 29-32. [CrossRef]

18. Donetsky, D.; Belenky, G.; S vensson, S.; Suchalkin, S. Minority carrier lifetime in type-II InAs/GaSb strained-layer superlattices and bulk HgCdTe materials. Appl. Phys. Lett. 2010, 97, 052108. [CrossRef]

19. Giard, E.; Taalat, R.; Delmas, M.; Rodriguez, J.-B.; Christol, P.; Ribet-Mohamed, I. Radiometric and noise characteristics of InAs-rich T2SL MWIR pin photodiodes. J. Eur. Opt. Soc. Rapid Publ. 2014, 9, 14022. [CrossRef]

20. Plis, E.; Kim, H.S.; Bishop, G.; Krishna, S.; Banerjee, K.; Ghosh, S. Lateral diffusion of minority carriers in nBn based type-II InAs/GaSb strained layer superlattice detectors. Appl. Phys. Lett. 2008, 93, 123507. [CrossRef]

21. Casias, L.K.; Morath, C.P.; Steenbergen, E.H.; Umana-Membreno, G.A.; Webster, P.T.; Logan, J.V.; Kim, J.K.; Balakrishnan, G.; Faraone, L.; Krishna, S. Vertical carrier transport in strain-balanced InAs/InAsSb type-II superlattice material. Appl. Phys. Lett. 2020, 116, 182109. [CrossRef]

22. Plis, E.; Myers, S.; Kutty, M.N.; Mailfert, J.; Smith, E.P.; Johnson, S.; Krishna, S. Lateral diffusion of minority carriers in InAsSb-based nBn detectors. Appl. Phys. Lett. 2010, 97, 123503. [CrossRef]

23. Rhiger, D.R.; Smith, E.P. Carrier transport in the valence band of nBn III-V Superlattice infrared detectors. J. Electron. Mater. 2019, 48, 6053-6062. [CrossRef]

24. Durlin, Q.; Perez, J.P.; Rossignol, R.; Rodriguez, J.B.; Cerutti, L.; Delacourt, B.; Rothman, J.; Cervera, C.; Christol, P. InAs/InAsSb superlattice structure tailored for detection of the full midwave infrared spectral domain. In Proceedings of the SPIE Quantum Sensing and NanoElectronics and Photonics XIV, San Francisco, CA, USA, 29 January-2 February 2017; Volume 10111, p. 1011112.

25. Tsai, C.-Y.; Zhang, Y.; Ju, Z.; Zhang, Y.-H. Study of vertical hole transport in InAs /InAsSb type-II superlattices by steady-state and time-resolved photoluminescence spectroscopy. Appl. Phys. Lett. 2020, 116, 201108. [CrossRef]

26. Tennant, W.E.; Lee, D.; Zandian, M.; Piquette, E.; Carmody, M. MBE HgCdTe technology: A very general solution to IR detection, described by "Rule 07", a very convenient heuristic. J. Electron. Mater. 2008, 37, 1406-1410. [CrossRef] 